\title{
ACTUALITÉ ET ÉPISTÉMOLOGIE
}

Jamais depuis l'entre-deux-guerres peut-être la question des sciences et de l'objectivité scientifique n'a été aussi présente dans l'actualité, entendue au sens banal du mot, qu'au cours des derniers mois. En France, par exemple, plusieurs quotidiens et divers magazines ont traité de l'affaire Social Text ou bien ont donné écho à la parution de l'ouvrage Impostures intellectuelles qui lui fait suite.

Rappelons les faits. La revue Social Text fit paraitre dans son numéro de l'été 1996 un article intitulé « Transgressing the boundaries. Towards a transformative hermeneutics of quantum gravity ${ }^{1} »$. Il était signé par un professeur de physique de l'université de New York, Alan Sokal. Aussitôt après, l'auteur dévoilait dans une autre revue qu'il s'agissait d'un canular visant à stigmatiser chez les intellectuels et les universitaires des États-Unis le recours à une philosophie des sciences, implicite ou explicite, caractérisée par une conception conventionnaliste ou culturaliste de l'objectivité et des présupposés relativistes ${ }^{2}$. Revendiquant un geste expérimental, Alan Sokal entendait ridiculiser les emprunts frauduleux faits aux sciences mathématiques et physiques dans la littérature qu'il attaquait, ou encore l'habillage d'inanités philosophiques au moyen de références complaisantes à des auteurs le plus souvent français. En octobre dernier, le même Alan Sokal, accompagné cette fois de Jean Bricmont, professeur de physique à l'université de Louvain, publiait en France Impostures intellectuelles, un bêtisier qui épinglait bon nombre de ces références obligées outre-Atlantique, multipliant les exemples d'emplois incontrôlés ou fautifs de conclusions scientifiques.

Les articles et le livre ont fait grand bruit. Ainsi, à la mi-janvier 1998, pouvait-on compter une demi-douzaine de sites sur le Web qui fournissaient directement ou indirectement les principales prises de positions ou

1. Social Text, 46-47, Spring-Summer 1996, p. 217-252.

2. Alan SoKaL, "A physicist experiments with cultural studies ", Lingua Franca, MayJune 1996, p. 62-64.

Revue de synthèse : $4^{\mathrm{e}} \mathrm{S} . \mathrm{n}^{\circ} 1$, janv.-mars 1998, p. 3-8. 
réactions, occasionnées par le canular et ses suites ${ }^{3}$. La portée planétaire du support d'information ne doit cependant pas tromper. Il s'agit concrètement d'une dizaine d'activistes ouvrant effectivement au retentissement de l'affaire, lus par environ 18000 internautes pour l'un de ces sites par exemple ${ }^{4}$. Des revues spécialisées aux États-Unis et la presse en Europe en rendirent compte, en particulier à la sortie du livre en librairie ${ }^{5}$.

La lecture des documents diffusés sur le Web suggère qu'au-delà de l'entretien de la polémique par ses principaux animateurs, d'une série de réponses fournies ici ou là et de très nombreuses recensions par des commentateurs, le phénomène auquel on a pu assister n'est pas à proprement parler un débat intellectuel ou scientifique. C'est, d'ailleurs, l'un des arguments de Sokal et de Bricmont que de réclamer l'examen effectif des analyses et des critiques que procure leur livre. L'hétérogénéité des interprétations accordées dans ce corpus au canular et à la dénonciation d'impostures intellectuelles est patente. L'attention méticuleuse des deux principaux auteurs à répondre à la plupart des prises de positions les plus autorisées, et le travail de recueil, de récapitulation de l'affaire, sans cesse réactualisé sur les sites Web, renforcent l'illusion d'un débat structuré alors qu'on assiste à des séries de réactions qui révèlent plus les variantes de la culture épistémologique contemporaine, principalement aux États-Unis et en France, qu'une véritable controverse intellectuelle.

Il est donc bien difficile aujourd'hui de tracer un état des lieux de l'« après Sokal », alors même que beaucoup parmi ceux qui étudient les sciences, de quelque manière que ce soit, ressentent qu'un tournant a été pris. Faute de pouvoir véritablement analyser le phénomène que constitue l'affaire virtuelle, faute même de disposer d'instruments pertinents pour saisir les conditions planétaires et technologiques de son retentissement, on peut constater que la provocation dans Social Text n'était pas vaine, qu'elle a touché son but affiché ${ }^{6}$. On peut aussi se demander si, porté par ce pre-

3. Notamment, à la mi-janvier 1998 : http://www.physics.nyu.edu/faculty/sokal/;

http://www.blarg.net/jwalsh/sokal/;

http://www.math.tohoku.ac.jp/ koroki/sokal/;

http://peccatte.rever.fr/sbpresse/sokalbricmontpresse.html ou les sites du Centre for Social Theory and Technology, Keele University et HNet (Humanities On Line). L'ensemble procure une bibliographie de plus d'une centaine d'articles publiés dans divers pays, principalement aux États-Unis et en France, et des copies de beaucoup d'entre eux.

4. 18318 visites depuis le 6 juin 1996 sur le site de Jason Walsh cité précédemment.

5. En France, par ex., Libération, Le Monde, Le Monde de l'éducation, La Recherche, Liber, Le Nouvel Observateur, Le Canard enchaîné, Marianne, Pour la Science, Sciences humaines, Sciences et avenir, Charlie Hebdo, Le Point (certains de ces journaux ont diffusé sur le Web un dossier thématique spécialisé). Ailleurs, notamment : Social Text, Dissent, Tikkun, The New York Review of books, le Times Literary Supplement, The Guardian weekly, La Stampa, le Corriere della sera, Il Sole 24 Ore, La Repubblica, L'Unità, Die Zeit.

6. Précisons que le terrain avait été préparé par la publication du livre de Paul R. Gross et Norman LevitT, Higher Superstition. The academic left and its quarrels with science, Baltimore, MD, Johns Hopkins University Press, 1994. 
mier succès, les auteurs des Impostures, en relevant des bévues indiscutables certes, mais aussi en exerçant une censure intellectuelle peu charitable à l'égard des expériences métaphoriques et conceptuelles ${ }^{7}$, ne prennent pas le risque de se laisser entraîner dans un jeu de démagogie scientifique. De la stigmatisation judicieuse d'une mode ou d'une vulgate philosophique, ils en viennent à la critique recevable d'auteurs souvent fautifs. Mais cette critique est reçue, comme en témoigne les recensions, à la manière d'un règlement de comptes administré par les sciences mathématiques et physiques aux disciplines littéraires, comme la restauration d'une philosophie des sciences somme toute assez simpliste, comme enfin une attaque contre les frivolités intellectuelles françaises, autant d'effets déraisonnables.

Nous sommes ici dans une zone ambiguë entre l'expression d'une controverse qui ne se forme pas et la manifestation d'un état critique de la culture épistémologique des scientifiques et des universitaires contemporains. Que faire dans ces conditions ? Loïc Wacquant prolonge l'expérience inaugurée par le canular paru dans Social Text en analysant les premières réactions de défense du post-modernisme qu'il a suscitées ${ }^{8}$. Jacques Derrida, pour ce qui le concerne, constate le dérisoire de l'affaire ${ }^{9}$. Jacques Bouveresse ironise sur les effets différés de l'abandon de l'exigence de précision chez les auteurs incriminés ${ }^{10}$. Ces trois réponses, divergentes mais toutes défendables, ont en commun de chercher à se dégager de cette ambiguité $^{11}$. Il faut encore aller plus loin dans la clarification.

Ainsi, mettre en évidence, par voie de provocation ou de raisonnement, la faiblesse de vulgates philosophiques et sociologiques relatives aux sciences ne peut remplacer l'analyse des conditions qui ont préparé ou qui assurent leur succès. Les lieux communs en la matière en ont remplacé d'autres antérieurs, scientistes, culturellement dominants jusqu'aux années 1970. Ils procèdent aujourd'hui de la mise en doute de l'idée com-

7. Amy Dahan-Dalmedico s'en inquiète dans « Rire ou frémir. De la raillerie des postmodernes au désir de contrôler la pensée », La Recherche, 304, déc. 1997, p. 11. La question du statut heuristique des métaphores est plus délicate que ne le laisse croire la plupart des prises de position sur cette affaire, voir Judith SCHLANGER, «Connaissance et métaphore », Revue de synthèse, t. 116, 4, oct.-déc. 1995, p. 579-592.

8. Loïc WACQUANT, «Les dessus de I' "Affaire Sokal". 1. Une parodie post-moderne en actes ", Liber, 30, mars 1997, p. 9-10 et «Les dessus de 1' "Affaire Sokal”. 2. Petit précis d'alchimie post-moderne », Liber, 31, juin 1997, p. 14-16.

9. Jacques Derrida, « Sokal et Bricmont ne sont pas sérieux », Le Monde, 20 nov. 1997.

10. Jacques Bouveresse, «Les sots calent », Le Monde de l'éducation, 255, janv. 1998, p. 54-55.

11. Tel était aussi le motif d'une des premières réunions organisées à Paris après la parution des articles de l'été 1996 : «Sciences humaines versus sciences dures. Entre canulars virtuels et mystifications réelles ", Laboratoire disciplinaire " Pensée des sciences ", École normale supérieure, animé par Charles Alunni, avec Bernard Besnier, Éric Brian, Gilles Châtelet, Pierre Caye, Alain Prochiantz, le 11 décembre 1996. 
mune qu'on s'est longtemps faite des sciences. Ce basculement de la culture scientifique est un phénomène proprement historique qui reste à étudier. Le constat de l'existence d'un genre académique révèle la formation sociale de spécialistes patentés, qu'il s'agisse de nouveaux commentateurs des sciences désireux de s'affranchir de références philosophiques antérieures, ou encore de scientifiques conduits à rationaliser avec cynisme les conditions contemporaines de l'exercice de leur activité. Dans ces conditions, la logique du renouvellement des philosophies spontanées des sciences relèvent plus d'une transformation culturelle propre aux milieux savants que d'innovations effectivement théoriques. Les faiblesses visées par Sokal dans son canular initial trouvent leur raison dans ce moment particulier de l'histoire de la culture scientifique de la deuxième moitié du $\mathrm{xx}^{\mathrm{e}}$ siècle. Traquer l'erreur chez les auteurs dont la réception aux ÉtatsUnis alimente ce mouvement culturel particulier relève alors plus du donquichottisme que d'un programme raisonné.

On peut aussi s'étonner, dans les textes touchant à cette affaire, de l'amalgame par lequel une philosophie des sciences "à la française » se trouve désignée, alors même qu'il serait difficile d'accorder ensemble et sur ce terrain les différents auteurs incriminés par Sokal et par Bricmont ${ }^{12}$. Or, précisément, la particularité des travaux de langue française en la matière est la présence ancienne de plusieurs traditions intellectuelles durables depuis le début du siècle en matière de philosophie des sciences, d'histoire des sciences et d'épistémologie. La Revue de synthèse, depuis sa création en 1900, fut l'un des lieux privilégiés de leur expression. Une connaissance même sommaire de ces travaux montre aisément qu'on est loin des cibles de la critique du jour. Or, ces traditions proviennent précisément de débats explicites et élaborés entre philosophes, scientifiques, historiens et littéraires tout au long du $\mathrm{xx}^{\mathrm{e}}$ siècle. Stigmatiser aujourd'hui les sciences de la culture depuis les sciences naturelles revient à escamoter cette genèse. Enrico Castelli Gattinara l'a étudiée, pour la période de l'entre-deux-guerres, dans sa thèse et dans l'ouvrage qui paraîtra dans les prochaines semaines. Dans les deux premiers articles de ce numéro, il prolonge son enquête en examinant sur quel terrain l'agenda de l'histoire des sciences s'est formé pendant cette période et jusqu'à quel point la rencontre avec les fondateurs des Annales s'est opérée.

L'article suivant illustre l'un des registres dans lesquels les questions touchant à l'épistémologie de l'écriture de l'histoire se posent aujourd'hui. Maurice Lagueux y examine de manière critique les thèses de plusieurs auteurs relativement à la narration historique, ce qui le conduit à une inter-

12. Les Impostures intellectuelles visent, en effet, Jacques Lacan, Julia Kristeva, Luce Irigaray, Bruno Latour, Jean Baudrillard, Gilles Deleuze et Félix Guattari, Paul Virilio et les nombreux commentateurs imprudents du théorème de Gödel. 
rogation sur les formes prises par la philosophie spéculative de l'histoire et à une réflexion sur le réalisme historique. Il faut bien en passer par là pour évaluer la portée philosophique des conclusions tirées par les historiens des sciences.

Il importe donc de se dégager des règlements de comptes de discipline à discipline, tant ils ne peuvent conduire qu'à l'affirmation tautologique de la scientificité de chaque science. C'est pourquoi, on ne peut échapper à l'examen historiographique et critique du destin de l'histoire des sciences et de l'épistémologie au $\mathrm{xx}^{\mathrm{e}}$ siècle. Le chantier est vaste. Il est indispensable à la réévaluation des agendas des enquêtes à venir. Il intéresse donc la Revue de synthèse au premier chef.

Or, c'est précisément dans ses colonnes, au fil du siècle, qu'on peut suivre, directement ou indirectement, l'itinéraire des travaux spécialisés. Une réponse concrète aux interrogations qui foisonnent aujourd'hui dans l'étude des sciences et dans la discussion de leurs présupposés consiste donc à créer un outil empirique d'investigation du corpus de la revue depuis 1900. C'est l'objet de la convention signée à l'automne 1997 entre la Fondation "Pour la science »-Centre international de synthèse, les éditions Albin Michel et la Bibliothèque nationale de France. Elle conduira à la numérisation intégrale de tous les numéros de la Revue de synthèse de 1900 à 1999, et à la livraison du même corpus sous forme électronique pour lequel la navigation et la recherche systématique seront facilitées ${ }^{13}$.

Si la démarche suivie par Sokal et par Bricmont provient d'une simplification abusive, si les procédés employés sont discutables, la cible première de leurs attaques, le relativisme épistémologique, n'en disparaît pas pour autant. Ici encore, les prises de positions induites par les conflits de disciplines peuvent escamoter un objet de recherche. On peut, en effet, s'interroger sur des configurations historiques plus anciennes qui s'apparentent à celle d'aujourd'hui. Les variantes antiques du pyrrhonisme, certaines formes prises par le scepticisme au XVII ${ }^{\mathrm{e}}$ siècle, la critique de la science officielle pendant la seconde moitié du Xix ${ }^{\mathrm{e}}$ siècle sont autant d'éléments de comparaison. Il reste à identifier d'autres formes analogues et à qualifier les rapports sous lesquels on peut les comparer aux mouvements actuels. Ce n'est pas dire qu'il faille admettre que les différentes expressions de ces doutes radicaux valent les uns pour les autres. Bien au contraire, d'une époque à l'autre, les conditions culturelles et intellectuelles du doute radical ont changé. Mais l'examen de ces conditions et l'évaluation de ces doutes

13. Voir Éric DusSERT, Yannick MAIGNIEN, « Numérisation et lecture savante. Le projet de collaboration avec le Centre international de synthèse autour de la Revue de synthèse " in Henri Berr et la culture du xx' siècle, Paris, Albin Michel, 1996, p. 293-299. Voir aussi Eric Dussert, Yannick MaIGnien, Jean-Didier W AGNeur, "Revues et hypertexte ", La Revue des revues, 22, 1997, p. 3-16. 
restent à entreprendre, ici encore, au titre de chantier de recherche. De même peut-on constituer comme objet d'enquête le travail de reprise opéré d'une époque à l'autre, en étudiant par exemple ce que les sceptiques et les savants de l'époque moderne concevaient du pyrrhonisme antique ${ }^{14}$. Or, on sait l'importance de ces formes de doutes dans la genèse du probabilisme latin par exemple, ou, bien sûr, dans celle de la science galiléenne et de la philosophie cartésienne. On mesure ici qu'une période caractérisée par l'empire du doute ne doit pas effrayer le vrai scientifique.

Comment comprendre alors l'ampleur prise par le canular de Sokal? On l'a vu, plusieurs tensions s'y conjuguent : des règlements de comptes entre sciences physiques et sciences sociales, des revendications philosophiques antagonistes et des conflits d'identités intellectuelles nationales ${ }^{15}$. Ce dernier élément fournit un indice. Assiste-t-on à un véritable débat qui traverserait l'Atlantique? Non, à l'évidence les enjeux aux États-Unis et en France ne sont pas les mêmes, les protagonistes non plus, pas plus que les médias qui portent les contributions. Ici, pour l'essentiel, une couverture de presse et un succès de librairie, là des débats dans les campus, dans des revues et une forte activité sur le Web. Toutefois, l'affaire Social Text est bien la première polémique scientifico-philosophique au temps de la mondialisation des échanges culturels. Pour apprécier sérieusement l'itinéraire d'un ouvrage, le va-et-vient d'une référence théorique, ou le destin d'un modèle intellectuel, il faudrait pouvoir disposer d'un cadre d'analyse adéquat à cette situation de fait. L'article d'Abram de Swaan fournit un tel cadre. Il examine les propriétés que doit satisfaire une sociologie des échanges culturels transnationaux contemporains, en propose un modèle et le précise dans le cas de la configuration mondiale du système des langues. La portée de la construction va bien au-delà du cas qui nous sert ici d'introduction : il s'agit de dépasser les bavardages sur la globalisation en indiquant un schéma analytique compatible avec une sociologie historique de l'État inspirée par Norbert Elias. À sa lecture, on en vient à concevoir que l'affaire qui nous occupe manifeste un processus de «créolisation » dont la logique générale est esquissée par de Swaan. Gardons-nous donc des réactions de «petits blancs » et saisissons sous les feux de cette actualité ce que le doute épistémologique comporte de plus actuel.

Éric BRIAN

14. Le prochain numéro de la Revue de synthèse, t. 119, 2-3, avr.-sept. 1998, traitera du scepticisme classique et de son historiographie.

15. Bruno Latour, l'une des principales cibles d'Alan Sokal, a réagi en ripostant sur ce dernier thème dans « Y a-t-il une science après la guerre froide? », Le Monde, 18 janv. 1997. 